\title{
Optical Neural Stimulation Modeling on Degenerative Neocortical Neural Networks
}

\author{
M. Zverev, F. Fanjul-Vélez, I. Salas-García, J. L. Arce-Diego \\ Applied Optical Techniques Group, TEISA Department, University of Cantabria, Av. de los Castros \\ $\mathrm{s} / \mathrm{n}, 39005$ Santander (Spain) \\ fanjulf@unican.es; arcedj@unican.es
}

\begin{abstract}
Neurodegenerative diseases usually appear at advanced age. Medical advances make people live longer and as a consequence, the number of neurodegenerative diseases continuously grows. There is still no cure for these diseases, but several brain stimulation techniques have been proposed to improve patients' condition. One of them is Optical Neural Stimulation (ONS), which is based on the application of optical radiation over specific brain regions. The outer cerebral zones can be noninvasively stimulated, without the common drawbacks associated to surgical procedures. This work focuses on the analysis of ONS effects in stimulated neurons to determine their influence in neuronal activity. For this purpose a neural network model has been employed. The results show the neural network behavior when the stimulation is provided by means of different optical radiation sources and constitute a first approach to adjust the optical light source parameters to stimulate specific neocortical areas.
\end{abstract}

Keywords: infrared neural stimulation, optical propagation, neurodegenerative disease, neural network.

\section{INTRODUCTION}

According to Eurostat, $18.2 \%$ of European population is aged or over 65 in 2013, and it is predicted that by 2080 this percentage will rise to $28.7 \%$, and thus more people will suffer from pathologies in nervous system [1]. Nowadays multiple techniques of nervous system stimulation have developed and studied, but the definite cure still has not been discovered. There are noninvasive techniques, like transcranial magnetic stimulation (TMS) or transcranial direct current stimulation (tDCS), that have been proven to be secure and efficient [2].

Optical radiation can stimulate outer deep brain regions noninvasively, but the stimulation of deeper regions requires a surgical intervention [2]. ONS stimulates brain regions via optical radiation and alters neural activity. This work is focused on analyzing the changes induced by these alterations. Due to the small size of the illuminating spot this technique is more selective than TMS. Specific brain regions with complex shapes can be stimulated using an array of lasers. It is important to know how the energy is distributed in the illuminated area, and how it affects brain activity. Section 2 shows the optical propagation model of ONS. In section 3 neural network model is described. Section 4 describes the modeling of stimulation. Section 5 contains the results and discussion of this work. Final conclusions are presented in the section 6 .

\section{OPTICAL NEURAL STIMULATION: LIGHT PROPAGATION}

Monte Carlo methods have been used to find the penetration depth of the optical radiation and how much energy is delivered to the brain tissue [3]. Specifically, open-source Mesh-based Monte Carlo method was used [4]. The method consists in launching packets of photons to a head structure and observing their trajectory and energy. During the propagation of a package multiple scattering events occur. Between the scattering events the energy of the photon package is attenuated as:

$$
\Delta W=\frac{\mu_{a}}{\mu_{e}} W
$$

being $W$ the package weight, that represents its energy, $\mu_{e}$ is the extinction coefficient and $\mu_{e}$ is the absorption coefficient of the medium. The polar angle of the scattered photon package is:

Advanced Microscopy Techniques IV; and Neurophotonics II, edited by Emmanuel Beaurepaire,

Peter T. C. So, Francesco Pavone, Elizabeth M. Hillman, Proc. of SPIE-OSA Vol. 9536, 95361।

(c) 2015 SPIE-OSA · CCC code: $1605-7422 / 15 / \$ 18 \cdot$ doi: $10.1117 / 12.2183472$ 


$$
\theta= \begin{cases}\operatorname{acos}\left\{\frac{1}{2 g}\left[1+g^{2}-\left(\frac{1-g^{2}}{1-g+2 g \xi_{2}}\right)^{2}\right]\right\}, & g \neq 0, \\ \operatorname{acos}\left(2 \xi_{2}-1\right) & , g=0\end{cases}
$$

where $g$ is the scattering anisotropy [5]. The pseudorandom number that defines the scattering events and their location is $\xi_{2}$, with uniform distribution between 0 and 1 . To ensure the accuracy of the results, the process of launching and propagating packages of photons is repeated $10^{6}$ times. The results are shown in Figure 1. It can be appreciated that optical illumination shows a high degree of spatial specificity. In addition, it was possible to observe that optical radiation is actually able to reach the cerebral cortex.
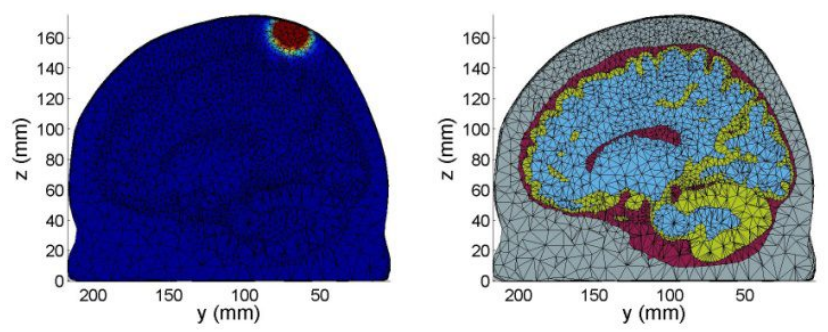

Figure 1. Results of optical propagation of a CW HeNe laser at $633 \mathrm{~nm}$. Optical radiation distribution for the sagittal plane at $\mathrm{x}=75.7 \mathrm{~mm}$. On the right, brain layer for the corresponding plane has been depicted.

\section{NEURAL NETWORK MODEL}

ONS stimulates a small region of neocortex, which is composed of a neural network that can be described by a mathematical model. The most known neuron model is the one described by Hodgkin and Huxley. This model can represent any neuron dynamic as it describes the action potential generation from the transmembrane ionic currents and membrane voltage [6]. In 2003 Izhikevich presented another neuron model [7]. It is simpler than the Hodgkin and Huxley's one and thus requires less calculus. In stead of describing the underlying phenomena of an action potential, the model properly mimics neuronal behavior omitting the transmembrane ionic currents. Therefore, the Hodgkin and Huxley model is appropriate to study different effects on a single neuron, but when it becomes necessary to model networks, the Izhikevich model will be more appropriate, as it gives realistic results and requires less calculus, and thus saves algorithm execution time. Izhikevich model is described by

$$
\begin{aligned}
& v^{\prime}=0.04 v^{2}+5 v+140-u+I \\
& u^{\prime}=a(b v-u) \text {, } \\
& \text { if } \quad v \geq 30 \mathrm{mV} \\
& \text { then }\left\{\begin{array}{c}
v \leftarrow c \\
u \leftarrow u+d
\end{array}\right.
\end{aligned}
$$

where $v$ is the membrane voltage of the neuron, $u$ is the membrane recovery variable, and $a, b, c$ and $d$ are constant parameters of the model and $I$ is the input current.

As the neocortex thickness is very small compared to its surface, $2.69 \mathrm{~mm}$ versus $1820 \mathrm{~cm}^{2}$ [8], in this work a twodimensional slice of a neocortical neural network is modeled. According to [8], the volumetric neuron density is around $44 \cdot 10^{6}$ neurons $/ \mathrm{cm}^{3}$, and the mean neocortical thickness is $2.69 \mathrm{~mm}$, which involves a surface neuron density of 118360 neurons $/ \mathrm{mm}^{2}$. The total number of neocortical neurons is around $21.5 \cdot 10^{9}$ neurons and the mean surface area is 1820 $\mathrm{cm}^{2}$, which give a slightly different value for surface neuron density, approximately 118132 neurons $/ \mathrm{mm}^{2}$. A 0.118 neurons $/ \mu \mathrm{m}^{2}$ surface neuron density is considered.

The modeled neural network is located in a $100 \mu \mathrm{m} \times 100 \mu \mathrm{m}$ neocortical slab, therefore it has 1180 uniformly distributed neurons. As in [7], the network receives thalamic input, the number of inhibitory neurons is $1 / 5$ of the total and the rest of the neurons are excitatory. B. Pakkenberg et al. in 2003 stated that on average, the neocortical neurons have about 7000 synapses each for intracortical reception and exchange of information [9]. This is much more than the number of 
neurons present in the model, thus all of the neurons are interconnected. The connections are defined as in [7], except for the fact that we did not connect neurons to themselves.

In neurodegenerative diseases the neurons suffer different kinds of defects and end up dying. The pathology modeled in this study randomly inactivates or kills $60 \%$ of neurons.

\section{STIMULATION MODEL}

The ONS effect is directly related to temperature increase induced by optical radiation absorption in the target brain region [10]. As the tissue absorbs energy, its temperature grows and stimulated neurons' membranes are depolarized. Therefore the depolarization can be assumed proportional to the absorbed energy. Thereby stimulation-induced depolarization is modeled as a membrane voltage increase in the stimulated neurons. This increase is proportional to the Gaussian function of the distance between the stimulated neuron and the central point of the stimulus incidence. The Gaussian function is scaled to make its maximum match the maximum depolarization. Different depolarization voltages have been tested to adjust the Gaussian stimulus. It has been observed that depolarizations greater than $47 \mathrm{mV}$ almost always cause a regular spiking (excitatory) neuron or a fast spiking (inhibitory) neuron to generate an action potential. In this study the maximum depolarization is set to $50 \mathrm{mV}$ and the Gaussian function's variance to $100 \mu \mathrm{m}^{2}$.

Complex neuophysiological parameters, such as the axon's length and width or the three-dimensional neural morphology, were modeled as white noise due to their random nature. This last one is then added to the stimulationinduced voltage and its standard deviation is $5 \%$ of the depolarization voltage.

The ONS is applied during very short time, in microseconds or picoseconds range. Thus, in order to properly model the stimulation, it would be necessary to use very small time steps in the microseconds or picoseconds range. However, taking into account that the action potential generation takes a few milliseconds and the time separation of the stimuli can be in the milliseconds range, the stimulation by a single ONS pulse can be modeled as a voltage increase in the right time step. The stimulation consists in 10 pulses repeated over different periods.

\section{RESULTS AND DISCUSSION}

The dynamics of the neural network described in section 3 has been computed for different cases. In the first case the network has no pathology whereas in the second one, the network has a pathology that randomly inactivates or kills $60 \%$ of the neurons. In the rest of the cases 10 stimulation pulses have been applied to the damaged network with periods of 30, 40, 50, 60 and $70 \mathrm{~ms}$. The network does not present synaptic plasticity. However, the modeled neurons adapt to activity of the connected neurons. Thus, it is possible to explore the network's response to stimulation by this neural activity plasticity. The neural activity stabilizes with time and the data of neural dynamics will be more stable if this dynamics computation time is longer. In order to observe the stimulation effects, it is necessary to avoid the complete neural dynamics stabilization. This is achieved by obtaining neural activity for 5 seconds $(5000 \mathrm{~ms})$. Figure 2 shows the dynamics of the first $1000 \mathrm{~ms}$. The graphics correspond to A) healthy network, B) damaged network with only $40 \%$ of active neurons and C) stimulated damaged network with a stimulation period of $40 \mathrm{~ms}$. In Figure $2 \mathrm{C}$ synchronous spikes induced by stimulation can be clearly appreciated. The last three groups of synchronous spikes are indicated by red arrows.
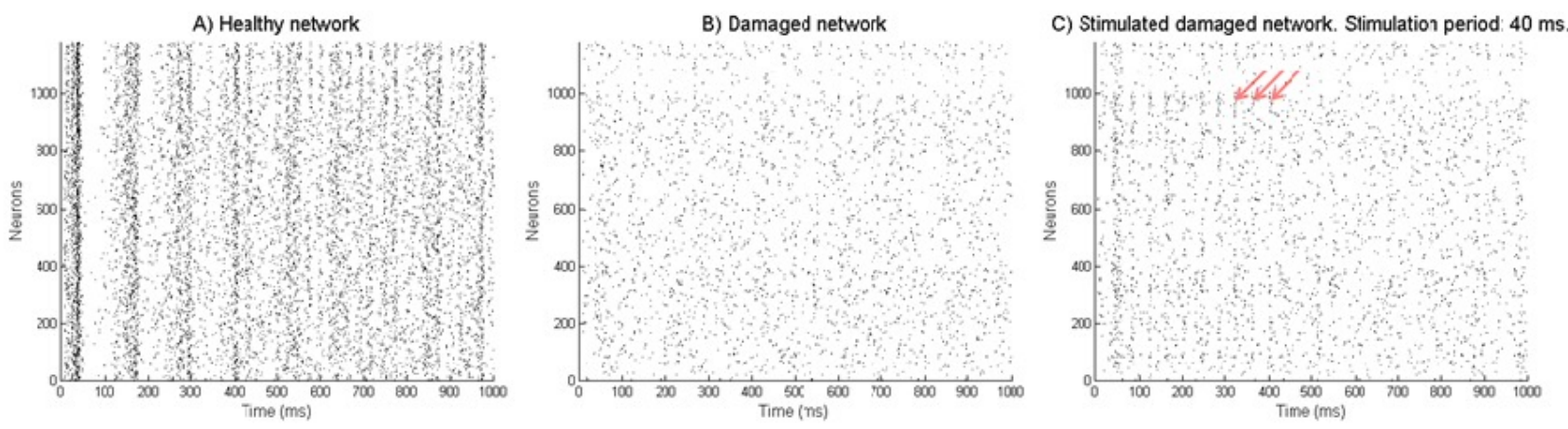

Figure 2. A) Dynamics of a healthy network. B) Dynamics of the same network with a pathology. $40 \%$ of neurons remain active. C) Dynamics of the stimulated damaged network. Stimulation period for this case was $40 \mathrm{~ms}$. 
The qualitative analysis of the spikes generated by each neuron can help to observe if the stimulation is applied and if it has any effect on the network. In order to properly evaluate the obtained data, spectral power at the frequency bands associated to the brain waves have been estimated for each studied case. The frequency bands considered in this work were $\delta, \theta, \alpha, \beta$ and $\gamma$. The spectral power associated to each of them was obtained after the last stimulation pulse and it is expressed as the percentage of the total spectral power in Figure 3A. Relative powers in $\delta$ band barely changes as depicted in Figure 3B.
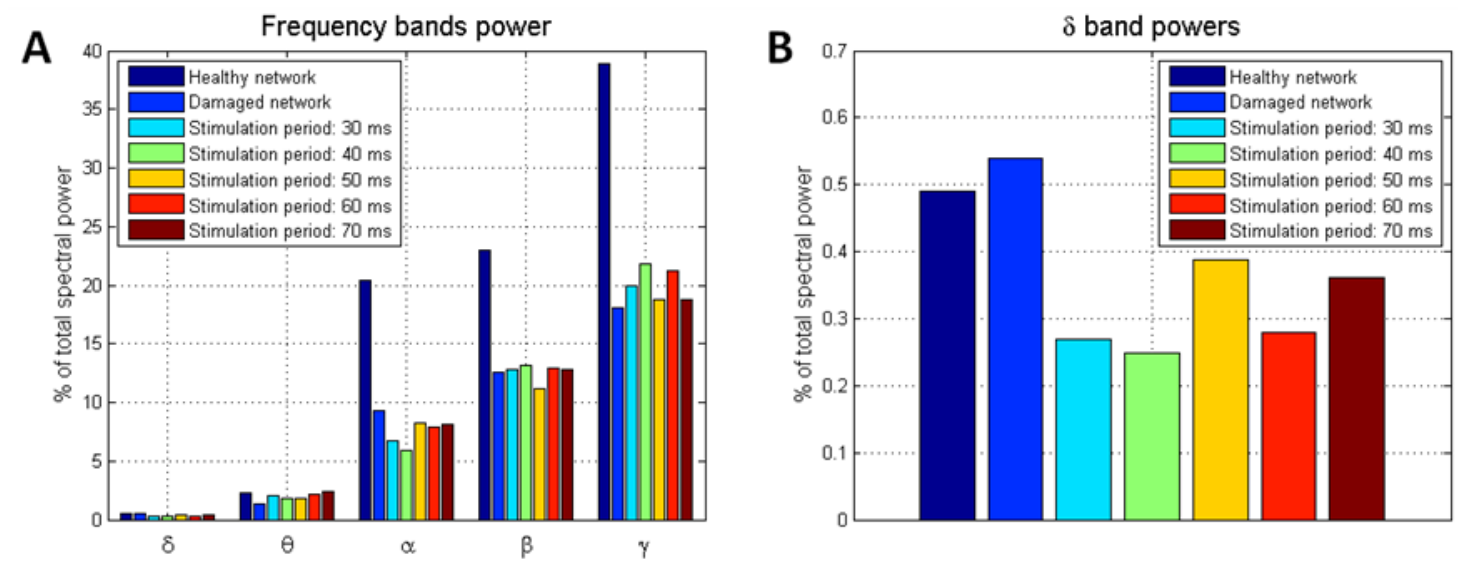

Figure 3. A) Spectral power of the frequency bands associated to brain waves generated by the modeled network. Data represented for healthy network, damaged network ( $40 \%$ active neurons) and stimulated damaged network, with stimulation periods of $30,40,50,60$ and $70 \mathrm{~ms}$. B) $\delta$ band spectral power.

The healthy network presents more power in the analyzed frequency bands than the damaged network even when the stimulation is applied. The damaged but not stimulated network has less relative power in its frequency bands. The difference is especially high in the $\alpha, \beta$ and $\gamma$ bands. The stimulated damaged network powers are similar to those of the non stimulated one.

When stimulation is applied, it can be seen that the relative power grows in the $\theta, \beta$ and $\gamma$ bands, except for the case of stimulation with $50 \mathrm{~ms}$ pulse separation, where the $\beta$ band power is lower than the non stimulated network power. In the $\gamma$ band the $50 \mathrm{~ms}$ stimulation power is also smaller than the rest of stimulated networks power, but it is higher compared to the non stimulated network band. In the alpha band all the studied stimulations decrease the relative power instead of increasing it. Although the $\delta$ band powers are very small, those in the damaged network increase whereas those in the stimulated network decrease to even lower values than in the healthy network (see Figure 3B).

\section{CONCLUSIONS}

In this work the ONS effects in stimulated neurons has been analyzed to determine their influence in neuronal activity. First optical radiation distribution in the cerebral neocortex was obtained. Then neural networks were modeled to study the impact of ONS on neural activity. The resulting model predicts the neural activity induced by optical stimulation and the obtained results constitute a first approach to determine the proper ONS parameters to induce specific changes in brain activity.

\section{ACKNOWLEDGMENTS}

This work has been partially supported by the project MAT2012-38664-C02-01 of the Spanish Ministry of Economy and Competitiveness, and by San Cándido Foundation. 


\section{REFERENCES}

[1] Statistical Office of the European Communities, "Population structure and ageing", EUROSTAT, May 2014, $<$ http://epp.eurostat.ec.europa.eu/statistics_explained/index.php/Population_structure_and_ageing > (20 May 2015).

[2] Fanjul-Vélez, F., Salas-García, I., Ortega-Quijano, N., and Arce-Diego, J. L., "FDTD-based Transcranial Magnetic Stimulation model applied to specific neurodegenerative disorders," Comput. Meth. Prog. Bio., 118(1), 34-43 (2015).

[3] Ortega-Quijano, N., Fanjul-Vélez, F., Salas-García, I. and Arce-Diego, J. L., "Numerical Modeling of Optical Radiation Propagation in a Realistic Model of Adult Human Head," Proc. IFMBE 41, 1697-1682 (2013).

[4] Fang Q., "Mesh-based Monte Carlo method using fast ray-tracing in Plücker coordinates," Biomed. Opt. Express, 32(1), 165-175 (2010).

[5] Wang, L. H., Jacques, S. L., Zheng, L. Q., "Monte Carlo modeling of light transport in multi-layered tissues," Comput. Meth. Prog. Bio., 47(2), 131-146 (1995).

[6] Hodgkin, A. L. and Huxley, A. F., "A Quantitative Description of Membrane Current and Its Application to Conduction and Excitation in Nerve," J. Physiol., 117(4), 500-544 (1952).

[7] Izhikevich, E. M., "Simple Model of Spiking Neurons," IEEE Trans. Neural Netw., 14(6), 1569-1572 (2003).

[8] Pakkenberg, B. and Gundersen, H. J. G., "Neocortical Neuron Number in Humans: Effect of Sex and Age," J. Comp. Neurol., 384(2), 312-320 (1997).

[9] Pakkenberg, B., Pelviga, D., Marnera, L., Bundgaarda, M. J., Gundersen, H. J. G., Nyengaardb, J.R., and Regeura, L., "Aging and the human neocortex," Exp. Gerontol., 38(1-2), 95-99 (2003).

[10] Wells, J., Kao, C., Konrad, P., Milner, T., Kim, J., Mahadevan-Jansen, A., and Jansen, E. D., "Biophysical Mechanisms of Transient Optical Stimulation of Peripheral Nerve,” Biophys. J., 93(7), 2567-2580 (2007).

[11] Norton, B. J., Bowler, M. A., Wells, J. D., and Keller M. D., "Analytical approaches for determining heat distributions and thermal criteria for infrared neural stimulation," J. Biomed. Opt., 18(9), 098001-1-098001-13 (2013). 